\title{
Driving Layer of Solar Activity
}

\author{
Jingxiu Wang \\ National Astronomical Observatories, Chinese Academy of Sciences \\ Beijing 100012, China
}

\begin{abstract}
There should be a driving layer on the Sun, in which the interaction between magnetic field and plasma motion would provide enough magnetic energy and necessary topology for the explosion of solar activity in the corona.

Although the exact location of the driving layer is not known, phenomenologically, the photosphere is acting, in many aspects, as the driving layer. Vector magnetic field measurements on the photosphere are greatly needed in clarifying the nature of the driving. Two elementary processes, flux emergence and cancellation, and one basic structure, magnetic interface between topology-independent magnetic loops, are key elements in the driving.
\end{abstract}

\section{Introduction}

Solar activity is defined as the explosive or catastrophic energy release in the solar atmosphere. It manifests as flare, filament eruption, and more global magnetic eruption, say coronal mass ejection (CME). They are powered by the magnetic energy, stored in the stressed magnetic fields in the solar atmosphere.

By driving it is meant how the magnetic fields in the solar atmosphere become stressed. Driving layer is a layer in which the interaction of magnetic field and plasma is most effective in putting magnetic energy and complexity into the higher atmosphere. Understanding the driving processes is critical for solar and space weather predictions.

\section{Conditions for the Driving Layer}

For an 'isolated' active region, the total magnetic energy changes in the 3-D volume can be written as a surface integration in the driving layer:

$$
\frac{d W}{d t}=\frac{1}{\mu} \int\left\{\left[\left(B_{\perp}^{2}-B_{z}^{2}\right) V_{z}-\left(\mathbf{V}_{\perp} \cdot \mathbf{B}_{\perp}\right) B_{z}\right]+\eta \mathbf{B}_{\perp} \cdot\left[\nabla B_{z}-\frac{\partial \mathbf{B}_{\perp}}{\partial z}\right]\right\} d x d y
$$

where, subscripts ' $z$ ' and $\perp$ refer to the longitudinal and transverse components. By familiar words in solar literature, the first term in the integration is due to flux emergence; the second, shearing of magnetic field by plasma motion; the third, Ohmic dissipation. 
To be effective in the driving, the following conditions should be satisfied. First, the magnetic field and plasma motion should be, more or less, independent, i.e. the the plasma $\beta$ should be close to 1 . Secondly, the plasma motion should be ordered in certain scales, and the field, organized collectively. If either of these are randomly distributed, there would be no energy storage to the atmosphere. Thirdly, a finite conductivity would favor the energy storage in the atmosphere by the surface Ohmic diffusion. Basically, the photosphere meets all the above requirements. In building-up energy, the plasma motion is as important as magnetic field. Moreover, the transverse magnetic field plays an important role in the driving. Therefore, the line-of-sight magnetic field alone can never provide complete information about the driving processes.

\section{Two Elementary Processes}

\subsection{Flux Emergence}

Emerging flux regions (EFRs) play a central role as drivers of solar activity. Their association with flares was well-established by the early 1980's (Švestka 1981). Further associations with filament eruption and CME's were identified later (Feynman and Martin 1995; Wang \& Sheeley 1999). EFRs appear to be a central agent in the creation of magnetic shear (Wang 1994).

The driving by EFRs is not due to their isolated input alone, but also, probably most importantly, by their interaction with pre-existing magnetic flux. This interaction always creates strongly curved magnetic neutral lines, often sigmoidal in shape, where there are steep gradients of $B_{z}$, strong magnetic shear, and continuous flaring and surging. With the flux emergence the related flares, EUV dimming and CMEs increase in extent (Wang and Shi 1992; Wang et al. 2000). This demonstrates that larger and larger volume of the active atmosphere become coupled and energized in flux emergence.

\subsection{Slow Reconnection in the Lower Atmosphere}

More and more examples have demonstrated that the gradual and mutual flux disappearance by flux cancellation is as important as, or even more important than flux emergence in driving solar activity. However, there have been diverse ideas in interpreting the observed flux cancellation, which are listed in Table 1.

Table 1. Interpretations of Flux Cancellation

\begin{tabular}{ll}
\hline Interpretation & Original Work \\
\hline R-loop Submergence & Parker (1987) \\
U-loop emergence & Spruit et al. (1987) \\
O-loop emergence & Lites et al. 1995 \\
Slow reconnection & Wang and Shi (1993) \\
& Roumeliotis and Moore (1993) \\
Reconnection submergence & Priest (1988) \\
\hline
\end{tabular}




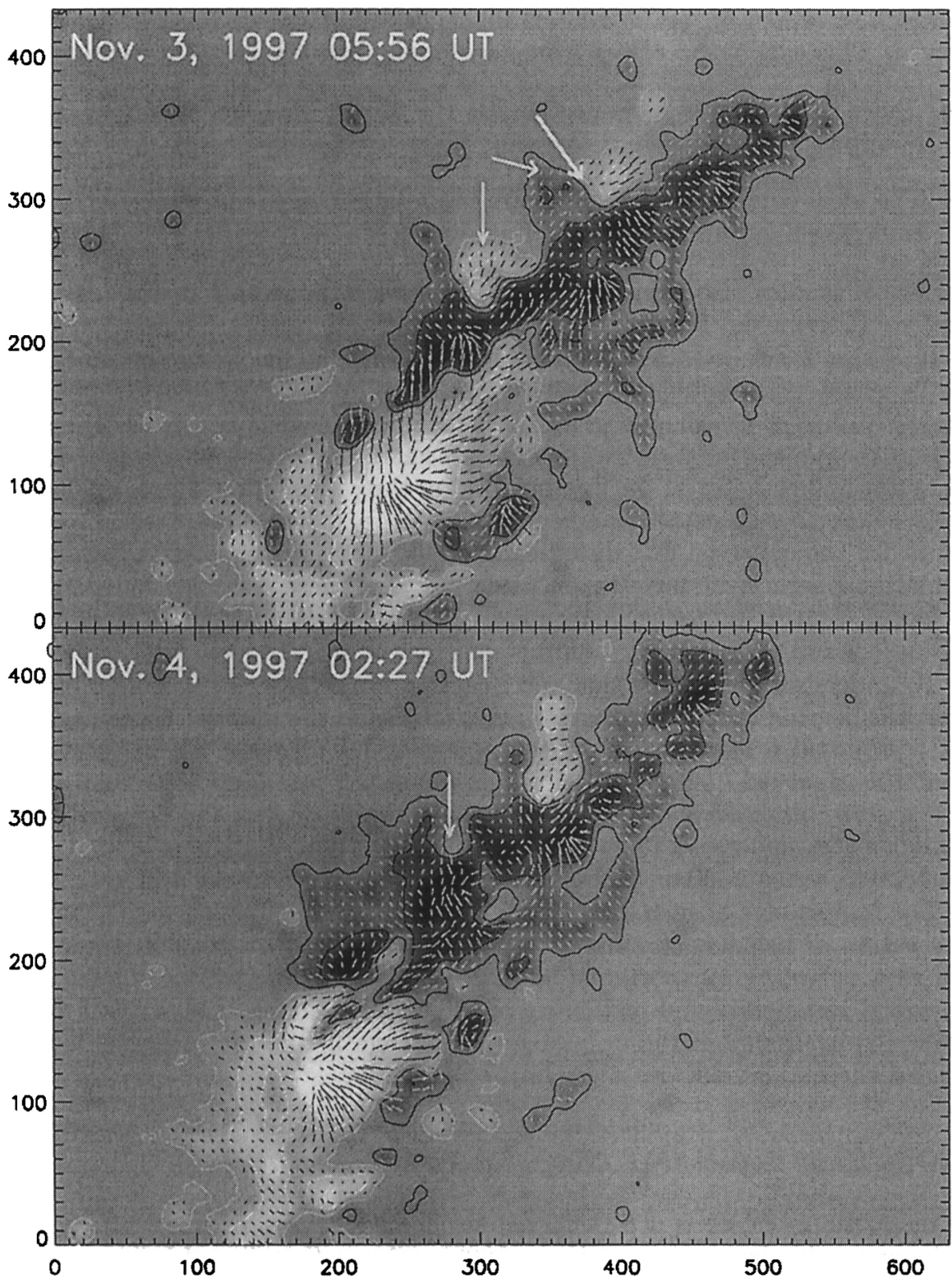

Figure 1. Two vector magnetograms of NOAA 8100 . The line-ofsight component is presented by brightness, with darker (lighter) color for negative (positive) polarity; the transverse components are presented by short line segments with their length proportional to field strength and alignment parallel to the field direction. The two left arrows indicate an EFR (EFR 1), and the right arrow marks the boundary of EFR 1 and another long-duration EFR (EFR 2). 
All of the proposed interpretations could be correct. But, at least, the magnetic reconnection either in, or below, or above the photosphere, must have been involved. This conclusion comes from two fundamental facts.

- There is no magnetic connection, or $\mathrm{H} \alpha$ fibrils, between two flux patches of opposite polarities in a canceling magnetic feature.

- The most commonly occurring flux cancellation takes place between newly emerging flux and pre-existing flux, which are not previously connected.

Theoretical studies also support the idea of slow reconnection in the lower atmosphere (Litvinenko 1999; Ji et al. 2000).

In Figure 1 we show an example of flux cancellation involving new emerging flux. In an interval of only one day, the positive flux of EFR 1 has disappeared completely, and at the magnetic interface (see Section 4) indicated by the long arrow in the figure, the negative flux from EFR 1 and other sources is reduced by a factor of 2 to 3 . Since the flux involved in the cancellation is not previously connected, neither $\Omega$-loop submergence, nor U-loop and O-loop emergence could account for the observed flux disappearance. The magnetic reconnection in the lower atmosphere must have taken place between these topology-independent flux loops. At this interface many flares took place. Among these were seven CME-associated (Wang et al. 2000).

To understand why such slow reconnection in the lower atmosphere has an important bearing on the explosive energy release in the corona, Wang and Shi (1993) proposed a two-step reconnection scenario for flares. Their basic idea is that the observed flux cancellation represents the first step of reconnection, which is slow, but continuous, in accumulating magnetic flux and complexity in the higher atmosphere. This aids in creating conditions for the second step of reconnection which is directly responsible for the energy release in flares.

The importance of such slow reconnections should be further exploited. By conservation of helicity, continuous reconnection in the lower atmosphere will serve as a primary transporter of helicity, and play an active role in creating topological complexity and instability in the corona. Zhang et al. (2000) found that flux cancellation is the only apparent change in the magnetic fields that preceded the major solar event on July 14, 2000.

\section{One Basic Structure - Magnetic Interface}

The magnetic interface is a 3-D curved surface where two or more interacting flux loops are in close contact (Wang, 1998). This definition naturally satisfies the definition of the quasi-separatrix layer (QSL) - a surface of field lines in close contact, which become widely separated when spreading out (Priest and Démoulin, 1995). The reason for choosing this term comes from the considerations that a separatrix, or QSL, is somehow a simplification of real magnetic interface between two topology-independent flux systems, and the identification of a separatrix depends on a priori assumption that all magnetic fields are free to connect other fields of opposite polarity. This is not true in solar atmosphere.

Magnetic interfaces can exist in the lower atmosphere. Their intersection with the photosphere may appear in an area of the same magnetic polarity, even 
within a single sunspot. As found by Yan and Wang (1995), a flare ribbon is, in most cases, a series of footpoints of several interacting loops, i.e., the site crossed by one or more magnetic interfaces.

If low-lying twisted EFR loops are the sources of energy in a magnetized atmosphere, the transfer if this energy takes place through the magnetic interface. In other words, the magnetic corona is continuously disturbed by the driver at the magnetic interface. The magnetic interface in the magnetized atmosphere is quite similar to the frontier surfaces between weather systems in the Earth's atmosphere.

\section{Concluding Remark}

In addition to newly emerging flux, slow reconnection in the photosphere or lower atmosphere appears to be a key element in driving solar activity. Magnetic interfaces are the primary sites of the driving. Although space observations have proven to be successful in monitoring the structure and dynamics of the solar corona, vector field observations in the photosphere serve as a key in solar prediction and space weather enterprise.

Acknowledgments. The work is supported by National Natural Science Foundation under Grants No.1979090, 19973009.

\section{References}

Feynman, J. \& Martin, S.F. 1995, J. Geophys. Res., 100(A3), 3355

Ji, H., Song, M. \& Huang, G. 2000, accepted by ApJ

Lites, B.W., Low, B.C., \& Martinez Pillet, V. et al. 1995, ApJ, 446, 877

Litvinenko, Y.E. 1999, ApJ, 515, 435

Parker, E.N. 1987, Solar Phys., 110, 11

Priest, E.R. \& Démoulin, P. 1995, J. Geophys. Res., 100, 23443

Priest, E.R. 1978, in E. Schröter et al. (eds.), The Role of Fine-Scale Magnetic Fields on the Structure of the Solar Atmosphere, Cambridge Univ. Press, 297

Roumeliotis, G. \& Moore, R.L. 1993, ApJ, 416, 386

Spruit, H.C., Van Ballegoijen, A.A., Title, A.M. 1987, Solar Phys., 110, 115

Švestka, Z. 1981, in E.R. Priest (ed.) Solar Flare Magnetohydrodynamics, 61

Wang, J. 1994, Solar Phys., 155, 285

Wang, J. 1998, ASP Conf. Series, 140, 121

Wang, J. \& Shi, Z. 1992, Solar Phys., 140, 67

Wang, J. \& Shi, Z. 1993, Solar Phys., 143, 119

Wang, J. et al. 2000, presented in IAU Symp. No. 203

Wang, Y.-M. \& Sheeley, M.R.Jr. 1999, ApJ, 510, L157

Yan, Y. \& Wang, J. 1995, A\&A, 298, 277

Zhang, J. et al. 2000, submitted to ApJ 